\title{
3,3-二甲基-二苯基-2,4-氧杂-8,10-氮杂螺[5.5]十一烷-1,5-二酮-9-硫酮 衍生物的有效合成
}

\author{
许招会*, $a$ 周 鹏 $b$ 刘德永 ${ }^{a}$ \\ ( ${ }^{a}$ 江西师范大学化学化工学院 南昌 330022) \\ ( ${ }^{b}$ 南昌理工学院新能源与环境工程学院 南昌 330029)
}

\begin{abstract}
摘要 在分子碘作用下，以芳香醛、硫艮和 2,2-二甲基-1,3-二噁烷-4,6-二酮为原料，通过无溶剂三组分缩合反应合成了 6 种 3,3-二甲基-二苯基-2,4-氧杂-8,10-氮杂螺 [5.5]十一烷-1,5-二酮-9-硫酮衍生物. 当催化剂的用量为 $10 \mathrm{~mol} \%$ 时, $80{ }^{\circ} \mathrm{C}$ 反应 $3.0 \sim 6.0 \mathrm{~h}$, 收率为 $73 \% \sim 91 \%$. 此外，该方法具有反应条件温和、后处理简单且收率高的优点.
\end{abstract}

关键词 分子碘; 无溶剂; 三组分反应; 螺杂双环化合物

\section{Efficient One-Pot Synthesis of 3,3-Dimethyl-diphenyl-2,4-dioxa- 8,10-diazaspiro[5.5]undecane-1,5-dione-9-thione Derivatives}

\author{
Xu, Zhaohui*,a $\quad$ Zhou, Peng $^{b} \quad$ Liu, Deyong ${ }^{a}$ \\ $\left({ }^{a}\right.$ College of Chemistry and Chemical Engineering, Jiangxi Normal University, Nanchang 330022) \\ ( ${ }^{b}$ School of New Energy and Enviroment Engineering, Nanchang Institute of Technology, Nanchang 330029)
}

\begin{abstract}
Six kinds of 3,3-dimethyl-diphenyl-2,4-dioxa-8,10-diazaspiro[5.5]-undecane-1,5-dione-9-thione derivatives were sythesized by the three component one-pot reaction of aromatic aldehydes with thiourea and 2,2-dimethyl-1,3-dioxane-4,6-dione in the presence of molecular iodine under solvent-free conditions. The results indicated that the yields ranged from $73 \%$ to $91 \%$ at $80{ }^{\circ} \mathrm{C}$ for $3.0 \sim 6.0 \mathrm{~h}$ using $10 \mathrm{~mol} \%$ iodine (relative to the substrate of 2,2-dimethyl-1,3-dioxane-4,6dione). Furthermore, the present procedure with high yields was milder conditions and simpler post-treatment process.
\end{abstract}

Keywords molecular iodine; solvent-free; three component condensation reaction; spiro hetrobicyclic compounds

3,3-二甲基-二苯基-2,4-氧杂-8,10-氮杂螺[5.5]十一 烷-1,5-二酮-9-硫酮系属螺杂双环衍生物, 是构成一些 含螺环结构天然化合物的结构单元 ${ }^{[1,2]}$. 由于在抗病毒、 抗肿瘤、降血压、临床麻醉表现出广泛生物或药理活 性 $^{[3,4]}$, 已引起人们的极大关注. 随着人们对该类杂环化 合物的深入研究和广泛应用, 发展和篮选具有新型结构 和低毒高效药理活性的螺杂双环衍生物, 已成为氧杂或 氮杂杂环化合物研究领域的热点. 3,3-二甲基-二苯基 -2,4-二氧杂-8,10-二氮杂螺 [5.5]十一烷-1,5,9-三酮作为 螺杂双环衍生物的典型代表, 一般由苯甲醛、尿素和 2,2-二甲基-1,3-二啞烷-4,6-二酮在碘 ${ }^{[5]} 、 N B S / A I B N^{[6]}$ 、 $\mathrm{TMSCl}^{[7]}$ 、磷铇钒杂多酸 ${ }^{[8,9]}$ 、纤维素磺酸 ${ }^{[10]}$ 、二苯基氨
基三氟甲磺酸离子液体 ${ }^{[11]}$ 等催化剂的催化作用或无催 化剂微波辐射三组分缩合而得 ${ }^{[12]}$. 由于反应时间长, 易 生成双取代缩合产物或 Michael 加成产物而降低反应收 率, 产物很难分离, 需要用过量的醛来减少副产物的生 成，因此发展绿色、简便和高效合成的新方法势在必行.

分子碘作为一种特殊的 Lewis 催化剂, 具有温和、 高效、环境友好、廉价易得及易于操作等优点. 它已广 泛用于绿色化学、药物合成及新型有机催化等领

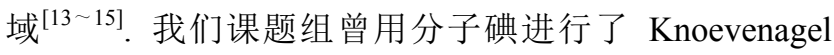
缩合 ${ }^{[16]}$ 及多组分反应 ${ }^{[17]}$ 反应, 发现其具有较好的催化 活性和高选择性. 基于此, 笔者发展了以分子碘为催化 剂，通过芳香醛、硫艮和 2,2-二甲基-1,3-二啞烷-4,6-二

* E-mail: gotoxzh@163.com

Received October 10, 2015; revised December 3, 2015; published online January 21, 2016.

Project supported by the National Science and Technology Project (No. 2001BA323C) and the Graduate Innovation Foundation of Jiangxi Province (No. YC2015-B023).

国家科技攻关计划(No. 2001BA323C)和江西省研究生创新基金(No. YC2015-B023)资助项目. 
酮(麦氏酸)三组分缩合反应合成 3,3-二甲基-二苯基2,4-氧杂-8,10-氮杂螺 [5.5]十一烷-1,5-二酮-9-硫酮衍生 物的简单、有效的方法. 合成路线见 Scheme 1.
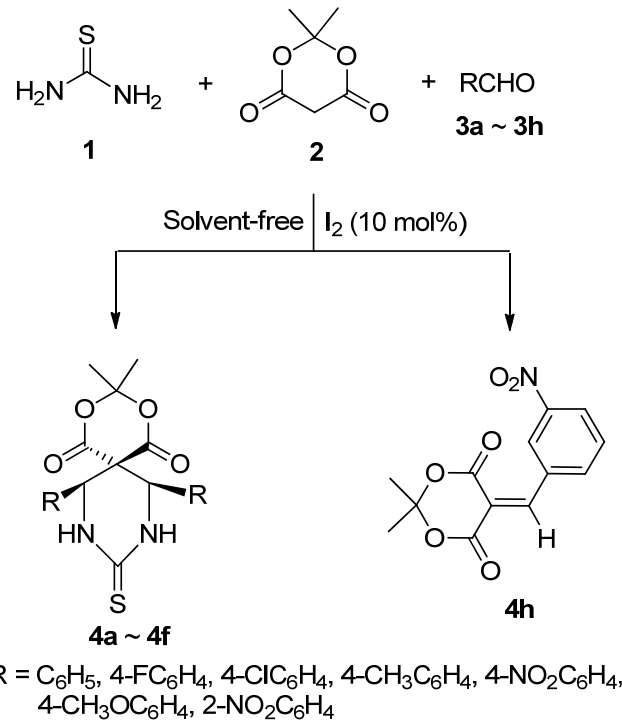

图式 1 化合物 $4 \mathrm{a} \sim 4 \mathrm{f}$ 和 $4 \mathrm{~h}$ 的合成

Scheme 1 Synthesis of $\mathbf{4 a} \sim \mathbf{4 f}$ and $\mathbf{4 h}$

\section{1 结果与讨论}

首先，以苯甲醛、硫脲和 2,2-二甲基-1,3-二啞烷-4,6二酮为反应模型，考察了不同溶剂、无溶剂、催化剂用 量、反应时间和反应温度对反应的影响, 其结果见表 1 . 从表 1 可以看出, 溶剂的极性对反应有一定的影响, 此 反应在极性溶剂中的收率高于非极性溶剂中的收率，但 在无溶剂条件下收率最高(表 1, Entries 1 9). 催化剂的 用量对反应的影响明显(表 1, Entries 9 14), 发现随着 催化剂用量的增加, 收率提高显著. 当催化剂用量为 10\%时，收率达到 $91 \%$, 继续增加催化剂用量达到 $12 \%$ 时, 收率仍为 $91 \%$. 当无催化剂时, 在相同反应条件下, 收率仅为 $54 \%$. 反应温度和反应时间对产物收率影响的 结果表明, 最佳反应温度为 $80{ }^{\circ} \mathrm{C}$, 最佳反应时间为 4.0 h. 综上所述, 优化的反应条件是: 在无溶剂条件下, 以 $10 \mathrm{~mol} \%$ 碘作为催化剂, 反应原料苯甲醛、硫脲、2,2-二 甲基-1,3-二噁烷-4,6-二酮的物质的量之比为 $2: 1: 1$, 反应温度为 $80{ }^{\circ} \mathrm{C}$, 最佳反应时间为 $4.0 \mathrm{~h}$.

产物 $4 \mathbf{a}$ 的结构经 IR, ${ }^{1} \mathrm{H}$ NMR 、 ${ }^{13} \mathrm{C}$ NMR 和 ESI-MS 分析确证. 在红外光谱中, 3200、3060 $\mathrm{cm}^{-1}$ 为 $\mathrm{NH}$ 特征 伸缩振动吸收峰; $1771 、 1734 \mathrm{~cm}^{-1}$ 为 $\mathrm{C}=\mathrm{O}$ 的伸缩振动 吸收峰; $1622 \mathrm{~cm}^{-1}$ 为 $\mathrm{C}=\mathrm{S}$ 的伸缩振动吸收峰. 核磁共 振氢谱中, 在 $\delta 8.97$ 出现 $\mathrm{NH}$ 的单峰; 在 $\delta 7.18 \sim 7.39$ 出现芳氢的多重峰; 在 $\delta 5.32$ 出现 $\mathrm{CH}$ 的单峰; 在 $\delta 0.50$ 出现 $\mathrm{CH}_{3}$ 的单峰. 核磁共振碳谱分析与其结构相吻
表 1 化合物 $4 \mathrm{a}$ 合成条件的优化 ${ }^{a}$

Table 1 Optimization of reaction conditions for the synthesis of $4 a$

\begin{tabular}{clcccc}
\hline Entry & \multicolumn{1}{c}{ Solvent } & $\mathrm{I}_{2}(\mathrm{~mol} \%)$ & Temp. $/{ }^{\circ} \mathrm{C}$ & Time/h & Yield $^{b} / \%$ \\
\hline 1 & $\mathrm{CH}_{3} \mathrm{CH}_{2} \mathrm{OH}$ & $\mathrm{I}_{2}(10)$ & Reflux & 6.0 & 76 \\
2 & $\mathrm{CH}_{3} \mathrm{OH}$ & $\mathrm{I}_{2}(10)$ & Reflux & 6.0 & 75 \\
3 & $\mathrm{CH}_{3} \mathrm{CO}_{2} \mathrm{CH}_{2} \mathrm{CH}_{3}$ & $\mathrm{I}_{2}(10)$ & Reflux & 6.0 & 70 \\
4 & $\mathrm{CH}_{3} \mathrm{CN}$ & $\mathrm{I}_{2}(10)$ & Reflux & 6.0 & 69 \\
5 & $1,4-\mathrm{Dioxane}$ & $\mathrm{I}_{2}(10)$ & Reflux & 6.0 & 62 \\
6 & $\mathrm{THF}$ & $\mathrm{I}_{2}(10)$ & Reflux & 6.0 & 64 \\
7 & $\mathrm{C}_{6} \mathrm{H}_{6}$ & $\mathrm{I}_{2}(10)$ & Reflux & 6.0 & 60 \\
8 & $\mathrm{CH}_{2} \mathrm{Cl}_{2}$ & $\mathrm{I}_{2}(10)$ & Reflux & 6.0 & 56 \\
9 & $\mathrm{None}_{10}$ & $\mathrm{I}_{2}(10)$ & 80 & 4.0 & 91 \\
11 & None & $\mathrm{I}_{2}(10)$ & 60 & 4.0 & 86 \\
12 & None & $\mathrm{I}_{2}(10)$ & 90 & 4.0 & 87 \\
13 & None & $\mathrm{I}_{2}(10)$ & 100 & 4.0 & 54 \\
14 & None & $\mathrm{I}_{2}(5)$ & 80 & 5.0 & 70 \\
15 & None & $\mathrm{I}_{2}(8)$ & 80 & 4.0 & 86 \\
\hline
\end{tabular}

${ }^{a}$ Reaction conditions: benzaldhyde $(10 \mathrm{mmol})$, thiourea $(5 \mathrm{mmol}), 2,2$-dimethyl-1,3-dioxane-4,6-dione (5 mmol), solvent $(15 \mathrm{~mL})$ or solvent-free; ${ }^{b}$ isolated yield.

合. ESI-MS 也证明了较强的 $397.1[\mathrm{M}+\mathrm{H}]$ 峰存在. 我们 培养了 $4 \mathbf{a}$ 的单晶并进行了 $X$ 射线单晶衍射, 图 1 为 $\mathbf{4 a}$ 的分子结构透视图. 在分子结构中两个芳香基团为顺式 结构, 与之相连接的六元环为呈扭椅式构象.

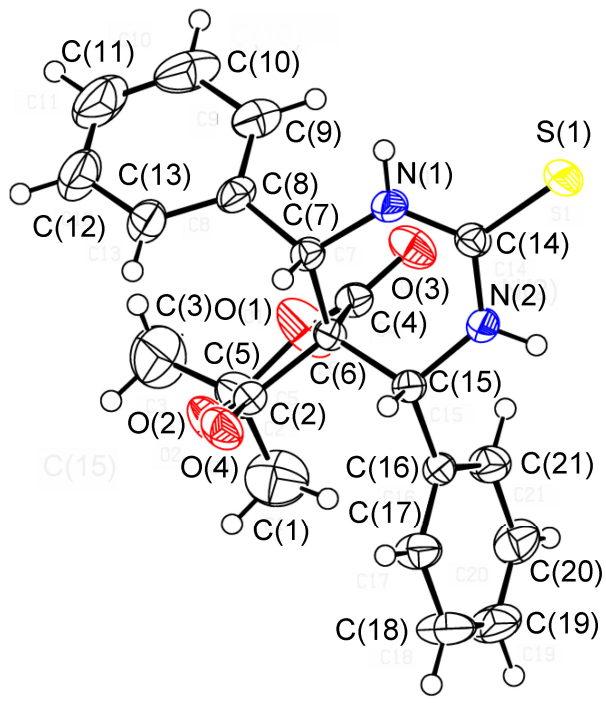

图 $14 \mathrm{a}$ 的分子结构透视图

Figure 1 X-ray crystal structure of $4 a$

接下来，在以上优化的反应条件下，取苯甲醛 10 $\mathrm{mmol}$ 、硫嫝 $5 \mathrm{mmol} 、 2,2$-二甲基-1,3-二啞烷-4,6-二酮 5 $\mathrm{mmol}$ 、催化剂用量 $10 \mathrm{~mol} \%$ 、反应温度为 $80{ }^{\circ} \mathrm{C}$ 、反应 时间为 $4.0 \mathrm{~h}$, 考察了碘、 $\mathrm{H}_{6} \mathrm{GeW}_{10} \mathrm{~V}_{2} \mathrm{O}_{40} 、 \mathrm{NBS} / \mathrm{AIBN}$ 及 对甲苯磺酸等催化剂对合成 3,3-二甲基-二苯基-2,4-氧 杂-8,10-氮杂螺[5.5]十一烷-1,5-二酮-9-硫酮的影响，实 验结果见表 2. 从表 2 可看出, $\mathrm{H}_{6} \mathrm{GeW}_{10} \mathrm{~V}_{2} \mathrm{O}_{40}$ 、对甲苯磺 
酸、NBS/AIBN 及乙酸的催化效果较差, 产品收率低, 分 子碘催化效果较好, 因此选择 $10 \mathrm{~mol} \%$ 分子碘作为最佳 催化剂.

表 2 不同催化剂的催化活性比较 ${ }^{a}$

Table 2 Comparison of catalytic activity of different catalyst

\begin{tabular}{clc}
\hline Entry & \multicolumn{1}{c}{ Catalyst $(\mathrm{mol} \%)$} & Yield $^{b} \%$ \\
\hline 1 & \multicolumn{1}{c}{-} & 54 \\
2 & NBS/AIBN (10) & 76 \\
3 & $4-\mathrm{CH}_{3} \mathrm{C}_{6} \mathrm{H}_{4} \mathrm{SO}_{3} \mathrm{H}$ & 65 \\
4 & $(10)$ & 80 \\
5 & $\mathrm{H}_{6} \mathrm{GeW}_{10} \mathrm{~V}_{2} \mathrm{O}_{40}(10)$ & 73 \\
6 & Acetic acid (10) & 91 \\
\hline
\end{tabular}

${ }^{a}$ Reaction conditions: aromatic aldehyde $(10 \mathrm{mmol})$, thiourea $(5 \mathrm{mmol})$, 2,2-dimethyl-1,3-dioxane-4,6-diones $(5 \mathrm{mmol})$, temperature $80{ }^{\circ} \mathrm{C}$, reaction time $4.0 \mathrm{~h}$, solvent-free conditions; ${ }^{b}$ isolated yield.

同时，为了探讨分子碘对该反应的普适性，在上述 优化反应条件下，通过改变不同芳香醛与硫艮、2,2-二 甲基-1,3-二噁烷-4,6-二酮进行三组分缩合反应，合成了 6 种螺杂双环衍生物 $\mathbf{4 a} \sim \mathbf{4 f}$. 从表 3 数据可以看出, 在 分子碤催化条件下, 芳香醛大多能反应均可以顺利进 行, 并以 73\% 91\%的高收率得到相应的 3,3-二甲基-二 苯基-2,4-氧杂-8,10-氮杂螺 [5.5]十一烷-1,5-二酮-9-硫酮 衍生物. 带吸电子基的芳香醛反应速度快于带供电子基 的芳香醛，对于带有强供电基团如 4-甲氧基苯甲醛也 能以 $73 \%$ 的收率得到目标产物. 另外在实验中发现对于 空间位阻较大的芳香醛如 2-硝基苯甲醛仅能发生 Knoevenagel 缩合反应.

表 3 化合物 $4 \mathrm{a} \sim 4 \mathrm{f}$ 和 $4 \mathrm{~h}$ 的合成 ${ }^{a}$

Table 3 Synthesis of $\mathbf{4 a} \sim \mathbf{4 f}$ and $\mathbf{4 h}$

\begin{tabular}{cclcc}
\hline Entry & Product & \multicolumn{1}{c}{$\mathrm{R}$} & Time/h & Yield $^{\mathrm{c}} \%$ \\
\hline 1 & $\mathbf{4 a}$ & $\mathrm{C}_{6} \mathrm{H}_{5}$ & 4.0 & 91 \\
2 & $\mathbf{4 b}$ & $4-\mathrm{FC}_{6} \mathrm{H}_{4}$ & 3.0 & 85 \\
3 & $\mathbf{4 c}$ & $4-\mathrm{ClC}_{6} \mathrm{H}_{4}$ & 3.5 & 88 \\
4 & $\mathbf{4 d}$ & $4-\mathrm{CH}_{3} \mathrm{C}_{6} \mathrm{H}_{4}$ & 5.0 & 76 \\
5 & $\mathbf{4 e}$ & $4-\mathrm{NO}_{2} \mathrm{C}_{6} \mathrm{H}_{4}$ & 3.0 & 83 \\
6 & $\mathbf{4 f}$ & $4-\mathrm{CH}_{3} \mathrm{OC}_{6} \mathrm{H}_{4}$ & 6.0 & 73 \\
7 & $\mathbf{4 h}$ & $2-\mathrm{NO}_{2} \mathrm{C}_{6} \mathrm{H}_{4}$ & 3.0 & 79 \\
\hline
\end{tabular}

${ }^{a}$ Reaction conditions: aromatic aldehyde $(10 \mathrm{mmol})$, thiourea $(5 \mathrm{mmol})$, 2,2-dimethyl-1,3-dioxane-4,6-diones $(5 \mathrm{mmol})$, iodine $10 \mathrm{~mol} \%$, temperature $80{ }^{\circ} \mathrm{C}$, solvent-free conditions; ${ }^{b}$ observed Knoevenagel reaction product; ${ }^{c}$ isolated yield.

此外, 根据产物的结构和反应的特点, 参考文献 [18，19]的方法，笔者认为苯甲醛的取代基效应对发生 三组分缩合反应可能存在不同的反应机理(Scheme 2). 对位取代苯甲醛 3a 3f 与硫䐂 1 在分子碘的催化下亲 核加成快速脱去一分子 $\mathrm{H}_{2} \mathrm{O}$ 而形成缩合产物硫酰基亚 胺中间体 5. 2,2-二甲基-1,3-二噁烷-4,6-二酮与硫酰基亚
胺中间体 5 发生 Michael 加成类似反应形成中间体 6. 中 间体 6 带部分正电荷的 $\alpha$-C，当芳环上有吸电子取代基 时，其 $\alpha-\mathrm{C}$ 的正电性增加，使反应速度加快，同时中间 体 6 继续与另一分子苯甲醛发生脲醛缩合反应，形成中 间体 7, 再发生 Michael 加成类似反应得目标产物 $\mathbf{4 a} \sim$ 4f. 2-硝基苯甲醛可能经过反应途径 B，在分子碘的催化 下通过 Knoevenagel 缩合反应快速脱去一分子 $\mathrm{H}_{2} \mathrm{O}$ 达到 目标产物 $\mathbf{4 h}$, 由于空间位阻的影响而不能与硫脲 $\mathbf{1}$ 进一 步发生 Michael 加成类似反应. 不过, 该机理还需要进 一步研究和论证。

\section{2 结论}

在无溶剂条件下采用碘催化不同芳香醛与硫脲和 2,2-二甲基-1,3-二噁烷-4,6-二酮发生三组分缩合反应, 有效合成了 6 种 3,3-二甲基-二苯基-2,4-氧杂-8,10-氮杂 螺 [5.5]十一烷-1,5-二酮-9-硫酮衍生物，并确定了较好的 反应条件：当反应原料苯甲醛、硫䏱与 2,2-二甲基-1,3二噁烷-4,6-二酮的物质的量之比为 $2: 1: 1$, 催化剂的 用量为 $10 \mathrm{~mol} \%$ 时, $80{ }^{\circ} \mathrm{C}$ 反应 $3.0 \sim 6.0 \mathrm{~h}$, 产品收率为 $73 \% \sim 91 \%$. 该方法不仅是对 Biginelli 三组分缩合反应 的重要拓展, 而且具有操作简单、反应时间短、收率高 等优点.

\section{3 实验部分}

\section{1 试剂与仪器}

2,2-二甲基-1,3-二噁烷-4,6-二酮按文献[20]制备; 苯甲醛、4-甲基苯甲醛、4-氯苯甲醛、4-氟苯甲醛、3氯苯甲醛、4-硝基苯甲醛、2-硝基苯甲醛及 4-甲氧基苯 甲醛为分析纯; 其它均为化学纯.

瑞士 BuchiB-540 型显微熔点仪(温度计未经校正); 德国 Bruker $400 \mathrm{MHz}$ 型核磁共振仪(DMSO- $d_{6}$ 为溶剂, TMS 为内标); 日本岛津 FT-IR-8400 型红外仪 ( $\mathrm{KBr}$ 压 片); MS 谱由 ABI 公司 API3200 三重四级杆质谱仪记 录; 单晶 X 射线衍射在 Siemens 公司 P4 型四圆衍射仪 上进行.

\subsection{3,3-二甲基-二苯基-2,4-氧杂-8,10-氮杂螺[5.5]十} 一烷-1,5-二酮-9-硫酮衍生物 $4 \mathrm{a} \sim 4 \mathrm{f}$ 的合成

在 $50 \mathrm{~mL}$ 三颈烧瓶中加入芳香醛 $10 \mathrm{mmol}$ 、硫艮 (0.38 g, $5 \mathrm{mmol}) 、 2,2$-二甲基-1,3-二噁烷-4,6-二酮 5 $\mathrm{mmol}$ 和磑催化剂 $10 \mathrm{~mol} \%$ (相对于 2,2-二甲基-1,3-二噁 烷-4,6-二酮的摩尔分数), 加热于 $80{ }^{\circ} \mathrm{C}$ 反应 $3.0 \sim 6.0 \mathrm{~h}$. 反应完毕, 加入 $5 \%$ 硫代硫酸钠水溶液 $20 \mathrm{~mL}$, 摚拌洗 涤、过滤，滤饼用 $20 \mathrm{~mL}$ 蒸馏水洗涤，乙酸乙酯重结晶, 干燥得目标化合物 $\mathbf{4 a \sim 4 f}$ 


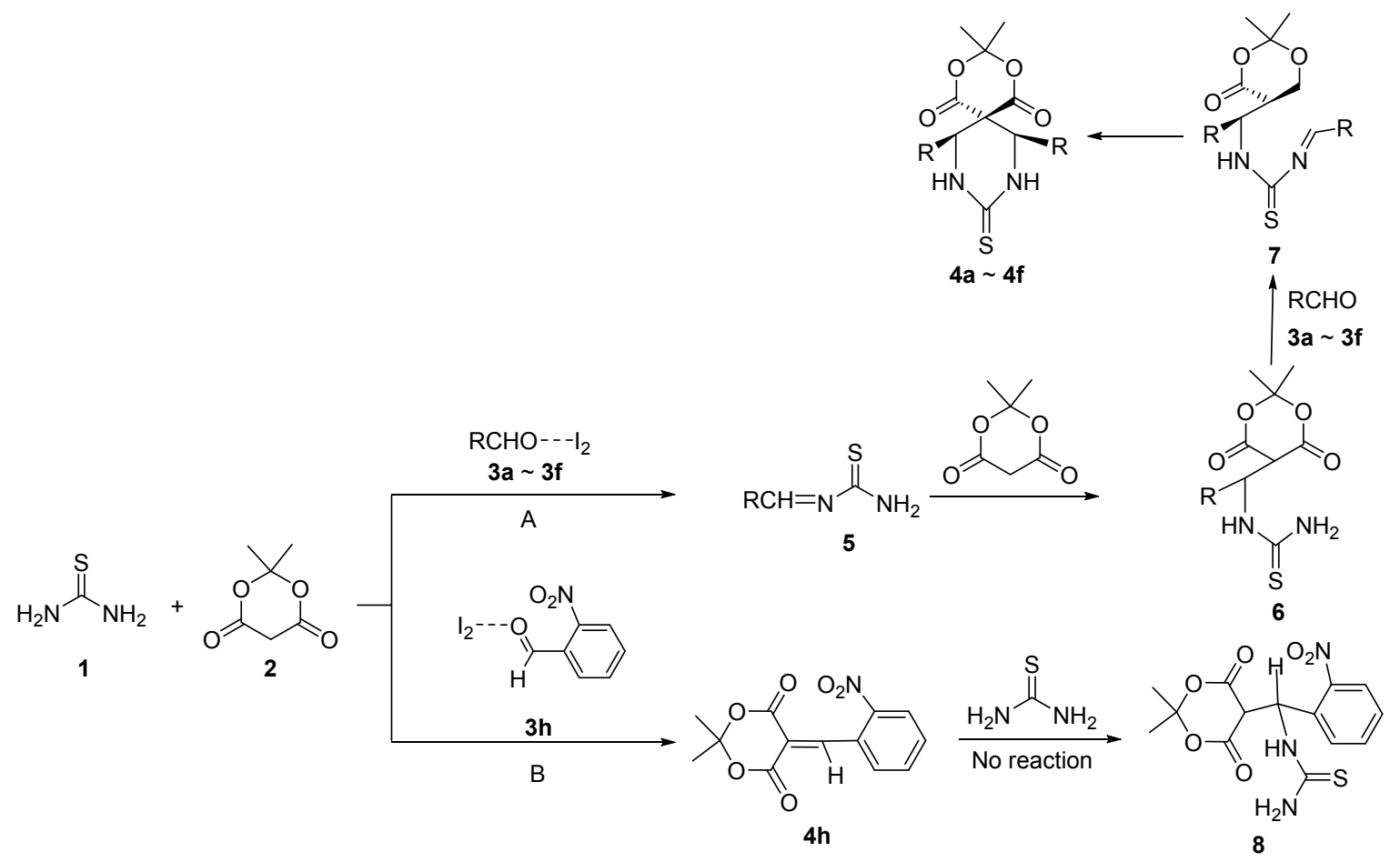

图式 2 碘催化下生成化合物 $\mathbf{4 a} \sim \mathbf{4 f}$ 和 $\mathbf{4 h}$ 的可能机理

Scheme 2 Possible mechanisms for the formation of $4 a \sim 4 f$ and $4 \mathrm{~h}$

3,3-二甲基-二苯基-2,4-氧杂-8,10-氮杂螺 [5.5]十一 烷-1,5-二酮-9-硫酮 $(\mathbf{4 a})$ : 白色固体，1.68 g, 收率 91\%. m.p. $204 \sim 206{ }^{\circ} \mathrm{C} ;{ }^{1} \mathrm{H}$ NMR (400 MHz, DMSO- $d_{6}$ ) $\delta$ : $0.50(\mathrm{~s}, 6 \mathrm{H}), 5.32(\mathrm{~s}, 2 \mathrm{H}), 7.18 \sim 7.39(\mathrm{~m}, 10 \mathrm{H}), 8.97$ (s, $2 \mathrm{H}, \mathrm{NH}) ;{ }^{13} \mathrm{C}$ NMR $\left(100 \mathrm{MHz}\right.$, DMSO- $\left.d_{6}\right) \delta: 28.15,56.84$ $\left(\mathrm{C}_{\text {spiro }}\right), 61.98,106.35,128.35,129.22,129.93,134.72$, 160.14, 165.74, 177.98; IR (KBr) v: 3200, 3060, 1770, 1734, 1653, $1209 \mathrm{~cm}^{-1}$; ESI-MS m/z: $397.1[\mathrm{M}+\mathrm{H}]^{+}$.

3,3 -二甲基-二(4-氟苯基)-2,4-氧杂- 8,10 -氮杂螺 [5.5]十一烷-1,5-二酮-9-硫酮(4b): 白色固体, $1.84 \mathrm{~g}$, 收 率 85\%. m.p. 208 210 ${ }^{\circ} \mathrm{C} ;{ }^{1} \mathrm{H}$ NMR (400 MHz, DMSO$\left.d_{6}\right) \delta: 0.61(\mathrm{~s}, 6 \mathrm{H}), 5.34(\mathrm{~s}, 2 \mathrm{H}), 7.19 \sim 7.30(\mathrm{~m}, 8 \mathrm{H}), 9.04$ (s, $2 \mathrm{H}, \mathrm{NH}) ;{ }^{13} \mathrm{C}$ NMR (100 MHz, DMSO- $\left.d_{6}\right) \delta: 28.30$, 56.88 ( $\left.\mathrm{C}_{\text {spiro }}\right), 61.25,106.45,116.05,116.27,130.59$, $130.68,130.86,130.89,160.27,161.83,164.28,165.67$, 177.93; IR (KBr) v: 3228, 3074, 1767, 1735, 1653, 1228 $\mathrm{cm}^{-1}$; ESI-MS $m / z: 433.1[\mathrm{M}+\mathrm{H}]^{+}$.

3,3-二甲基-二(4-氯苯基)-2,4-氧杂-8,10-氮杂螺 [5.5]十一烷-1,5-二酮-9-硫酮 (4c): 白色固体, $2.04 \mathrm{~g}$, 收 率 88\%. m.p. 208 $209{ }^{\circ} \mathrm{C}$; ${ }^{1} \mathrm{H}$ NMR $(400 \mathrm{MHz}$, DMSO- $\left.d_{6}\right) \delta: 0.62(\mathrm{~s}, 6 \mathrm{H}), 5.35(\mathrm{~s}, 2 \mathrm{H}), 7.18(\mathrm{~d}, J=8.0$ $\mathrm{Hz}, 4 \mathrm{H}), 7.51$ (d, $J=8.0 \mathrm{~Hz}, 4 \mathrm{H}), 9.08$ (s, $2 \mathrm{H}, \mathrm{NH}) ;{ }^{13} \mathrm{C}$ NMR (100 MHz, DMSO- $\left.d_{6}\right) \delta: 28.29,56.62$ ( $\left.\mathrm{C}_{\text {spiro }}\right), 61.26$, $106.52,129.27,130.29,133.57,134.60,160.11,165.52$,
177.96; IR (KBr) v: 3220, 3067, 1767, 1735, 1595,1296 $\mathrm{cm}^{-1}$; ESI-MS $m / z: 465.0[\mathrm{M}+\mathrm{H}]^{+}$.

3,3 -二甲基-二(4-甲基苯基)-2,4-氧杂- 8,10 -氮杂螺

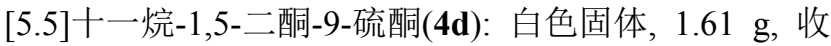
率 76\%. m.p. $222 \sim 224{ }^{\circ} \mathrm{C} ;{ }^{1} \mathrm{H}$ NMR $(400 \mathrm{MHz}$, DMSO- $\left.d_{6}\right) \delta: 0.54(\mathrm{~s}, 6 \mathrm{H}), 2.27(\mathrm{~s}, 6 \mathrm{H}), 5.24(\mathrm{~s}, 2 \mathrm{H}), 7.06$ (d, $J=8.0 \mathrm{~Hz}, 4 \mathrm{H}), 7.20(\mathrm{~d}, J=8.0 \mathrm{~Hz}, 4 \mathrm{H}), 8.87(\mathrm{~s}, 2 \mathrm{H}$, $\mathrm{NH}) ;{ }^{13} \mathrm{C}$ NMR (100 MHz, DMSO- $\left.d_{6}\right) \delta: 21.16,28.21$, $56.88\left(\mathrm{C}_{\text {spiro }}\right), 61.77,106.30,128.20,129.37,129.65$, $131.72,139.38,160.21,165.86,177.89$; IR (KBr) v: 3148, 3049, 1769, 1735, 1554, $1204 \mathrm{~cm}^{-1}$; ESI-MS $m / z: 425.1$ $[\mathrm{M}+\mathrm{H}]^{+}$.

3,3-二甲基-二(4-硝基苯基)-2,4-氧杂- 8,10 -氮杂螺 [5.5]十一烷-1,5-二酮-9-硫酮 (4e): 淡黄色固体, $2.01 \mathrm{~g}$, 收率 83\%. m.p. 232 $234{ }^{\circ} \mathrm{C} ;{ }^{1} \mathrm{H}$ NMR $(400 \mathrm{MHz}$, DMSO- $\left.d_{6}\right) \delta: 0.57$ (s, 6H), 5.59 (s, 2H), 7.47 (d, $J=8.0$ $\mathrm{Hz}, 4 \mathrm{H}), 8.32$ (d, $J=8.0 \mathrm{~Hz}, 4 \mathrm{H}), 9.34(\mathrm{~s}, 2 \mathrm{H}, \mathrm{NH}) ;{ }^{13} \mathrm{C}$ NMR (100 MHz, DMSO- $\left.d_{6}\right) \delta: 27.38,55.15$ ( $\left.\mathrm{C}_{\text {spiro }}\right), 60.17$, $105.78,122.97,123.29,129.07,129.29,140.61,147.59$, 158.84, 164.07, 176.94; IR (KBr) v: 3220, 3084, 1767, 1740, 1607, $1285 \mathrm{~cm}^{-1}$; ESI-MS $m / z: 487.1[\mathrm{M}+\mathrm{H}]^{+}$.

3,3 -二甲基-二(4-甲氧基苯基)-2,4-氧杂- 8,10 -氮杂 螺[5.5]十一烷-1,5-二酮-9-硫酮(4f): 淡黄色固体, $1.66 \mathrm{~g}$, 收率 73\%. m.p. $188 \sim 190{ }^{\circ} \mathrm{C} ;{ }^{1} \mathrm{H}$ NMR $(400 \mathrm{MHz}$, 
DMSO- $\left.d_{6}\right) \delta: 0.60(\mathrm{~s}, 6 \mathrm{H}), 3.73(\mathrm{~s}, 6 \mathrm{H}), 5.22(\mathrm{~s}, 2 \mathrm{H}), 6.96$ (d, $J=8.0 \mathrm{~Hz}, 4 \mathrm{H}), 7.09$ (d, $J=8.0 \mathrm{~Hz}, 4 \mathrm{H}), 8.84$ (s, 2H, $\mathrm{NH}) ;{ }^{13} \mathrm{C}$ NMR (100 MHz, DMSO- $\left.d_{6}\right) \delta: 27.23,54.68$, $56.00\left(\mathrm{C}_{\text {spiro }}\right), 60.46,105.20,113.13,113.48,125.41$, 128.50, 159.34, 159.42, 164.89, 176.76; IR (KBr) v: 3205, 3060, 1766, 1734, 1653, $1247 \mathrm{~cm}^{-1}$; ESI-MS $m / z: 457.1$ $[\mathrm{M}+\mathrm{H}]^{+}$.

5-(2-硝基苯基亚甲基)-2,2-二甲基-1,3-二噁烷-4,6二酮 $(\mathbf{4 h})$ : 淡黄色固体, $1.09 \mathrm{~g}$, 收率 79\%. m.p. 116 $117{ }^{\circ} \mathrm{C} ;{ }^{1} \mathrm{H}$ NMR (400 MHz, DMSO- $\left.d_{6}\right) \delta: 8.78$ (s, $\left.1 \mathrm{H}\right)$, $8.30(\mathrm{~d}, J=8.30 \mathrm{~Hz}, 1 \mathrm{H}), 7.75$ (t, $J=7.48 \mathrm{~Hz}, 1 \mathrm{H}), 7.61$ (d, $J=7.90 \mathrm{~Hz}, 1 \mathrm{H}), 7.50(\mathrm{~d}, J=7.50 \mathrm{~Hz}, 1 \mathrm{H}), 1.81(\mathrm{~s}$, $6 \mathrm{H}) ;{ }^{13} \mathrm{C}$ NMR (100 MHz, DMSO- $d_{6}$ ) $\delta: 28.12,105.69$, 117.42 , 124.33, 129.60, 129.88, 130.46, 133.47, 145.96, 154.85, 161.02; IR (KBr) v: 2965, 2925, 1762, 1737, 1640, $1604,1524,1380,1350,725 \mathrm{~cm}^{-1}$.

\section{$3.3 X$ 射线衍射实验}

晶体结构的测定在 Siemens P4 型四圆衍射仪上进 行. 将 $0.56 \mathrm{~mm} \times 0.54 \mathrm{~mm} \times 0.42 \mathrm{~mm}$ 白色棱形单晶置于 四圆衍射仪上, 用石墨单色器单色化的 Mo $\mathrm{K} \alpha(\lambda=$ $0.071073 \mathrm{~nm}$ )辐射, 以 $\omega / 2 \theta$ 扫描方式在 $1.71^{\circ}<\theta<$ $26.00^{\circ}$ 范围内收集个 4079 衍射点, 其中独立衍射点 3279 个 $\left(R_{\mathrm{int}}=0.0137\right)$, 可观测衍射点 1633 个 $[I>2 \sigma(I)]$.

辅助材料(Supporting Information) 目标化合物 $4 \mathbf{a} \sim$ $4 \mathbf{f}$ 的 ${ }^{1} \mathrm{H}$ NMR、 ${ }^{13} \mathrm{C}$ NMR 图谱. 这些材料可以免费从本 刊网站(http://sioc-journal.cn/)上下载.

\section{References}

[1] Rovnyak, G. C.; Kimball, S. D.; Barbara, B.; Gabriella, C.; John, D.
D.; Jack, G.; Anders, H.; Mary, M.; James, P. M. J. Med. Chem. 1995, 38, 19.

[2] Snider, B. B.; Shi, Z. J. Org. Chem. 1993, 58, 3828.

[3] Arimoto, H.; Hayakawa, I.; Kuramoto, M.; Uemure, D. Tetrahedron Lett. 1998, 39, 861.

[4] Chou, T.; Kuramoto, M.; Otani, Y.; Shkano, M.; Yazawa, K.; Uemere, D. Tetrahedron Lett. 1996, 37, 3867.

[5] Dipak, P.; Debajyoti, B.; Mukut, G.; Hu, W. H. Mol. Diversity 2011, $15,257$.

[6] Srinivasa, R. J.; Divya, V.; Shubha, J. J. Chem. Pharm. Res. 2012, 4, 2373.

[7] Zhu, Y. L.; Huang, S. L.; Pan, Y. J. Eur. J. Org. Chem. 2005, 2354.

[8] Srinivasa, R. J.; Divya,V.; Shubha, J. J. Catal. 2013, 2013, 1.

[9] Xu, Z. H.; Tu, Y. H. Chin. J. Org. Chem. 2015, 35, 1357 (in Chinese). (许招会, 涂缘鸿, 有机化学, 2015, 35, 1357.)

[10] Naser, M.; Khalil, P.; Masoud, B.; Soudabeh, K. Asian J. Chem. 2013, 25, 3373.

[11] Li, J. J.; Sun, J.; Su, W. K. Lett. Org. Chem. 2010, 7, 14.

[12] Dhevalapally, B. R.; Carlos, F. B. Chem. Eur. J. 2004, 10, 5323.

[13] Dohi, T.; Kita, Y. Chem. Commun. 2009, 28, 2073.

[14] Xu, W. J.; Zhang, W. S.; Yan, J. L. Chin. J. Appl. Chem. 2011, 28, 1143 (in Chinese). (许文静，张文龙，间金龙，应用化学，2011，28，1143.)

[15] Wang, D. L.; Dong, Z.; Liu, Z.; Yang, F. F. Chin. J. Org. Chem. 2014, 34, 783 (in Chinese). (王道林，董哲，刘忠，杨菲菲，有机化学，2014，34, 783.)

[16] Yan, N.; Xu, Z. H.; Liu, B. Chin. J. Spec. Petrochem. 2010, 27, 45 (in Chinese). (严楠，许招会，刘百强，精细石油化工, 2010, 27, 45.)

[17] Xu, Z. H.; Lin, C. H.; Liao, W. L. Chin. J. Appl. Chem. 2015, 32, 278 (in Chinese). (许招会, 林春花, 廖维林, 应用化学, 2015, 32, 278.)

[18] Bandgar, B. P.; Shaikh, K. A. Tetrahedron Lett. 2003, 44, 1959.

[19] Ahmad, S.; Ayoob, B.; Hamid, R. B. Mol. Diversity 2004, 8, 141.

[20] Yan. N.; Xiong, B.; Liao, W. L.; Xu, Z. H. Chin. J. Org. Chem. 2010, 30, 1391 (in Chinese). (严楠，熊斌，廖维林，许招会，有机化学, 2010, 30, 1391.) 